\title{
From the ASCO President
}

\section{Allied in Achieving the Best-Quality Cancer Care}

The Cancer Quality Alliance represents a major achievement in bringing together key stakeholders to coordinate the implementation of high-quality cancer care and to devise practical efforts for improvement. The Alliance's priorities are to develop a blueprint of quality oncology care, to adopt and test quality measures, and to identify the critical function and elements of an oncology electronic health record for practice, quality monitoring, and improvement. The Alliance will also focus on novel approaches to coordination of care and strategies for effective reimbursement for performance.

Quality cancer care means delivering the highest standards of management to oncology patients in all practice settings. The definition of quality - the "what" —is an essential first step, but the process—-the "how"-is especially daunting in our diverse health care system. The Alliance provides a forum for exchange of ideas, prioritizing and harmonizing efforts, and progressing more rapidly to practical implementation. Importantly, the Alliance can provide guidance with a unified voice to the Centers for Medicare \& Medicaid Services (CMS) as it moves toward a goal of payment linked to quality performance. But of greater importance, the activities of the Alliance provide necessary assistance for oncologists to reach their goal of delivering the best possible care to cancer patients.

My 2005-2006 ASCO presidential theme, "Advocating Survivorship, Clinical Science, and Oncology Quality Care," includes quality cancer care, an aspiration that unifies all ASCO membership-academic and community based, domestic and international, medical and all other oncology specialties. Multiple external forces converge in the pursuit of quality, including the rising expense of health care, reimbursement linked to performance, clinical trials, survivorship, and the ethics of appropriate use of resources and equity in cancer care. Recently, the cost of new cancer therapies has been highlighted in the media. Although cancer drugs have been under the spotlight, rising costs represent use of diagnostics, supportive care, and radiation and surgical oncology treatments. Oncologists have the opportunity, indeed the responsibility, to practice stewardship of limited health care resources in the choices they make. A blueprint for quality and practical implementation strategies are needed.

ASCO is uniquely poised to make major contributions to this national effort as leaders in defining and promoting the achievement of best outcomes. ASCO's National Initiative on Cancer Care Quality (NICCQ), the first ASCO program to measure the quality of cancer care, represents the vision of former ASCO President, Joseph Bailes, MD. Foremost among those areas in need of improvement identified by the NICCQ study was documentation. Under the leadership of Deborah Shrag, MD, ASCO is developing templates to summarize chemotherapy and biologic therapies delivered by medical oncologists. ASCO and National Cancer Centers Network (NCCN) expert panels recently selected colorectal and breast quality measures for full specification, pilot testing, and wide dissemination.

The Quality Oncology Practice Initiative (QOPI), ASCO's practice-based system of quality selfassessment, has been approved by the American Board of Internal Medicine as the only oncology-specific mechanism to qualify for performance assessment for maintenance of certification. QOPI has been expanded to all ASCO members and can serve as a testing ground for quality measures and their implementation. The Quality Advisory Group established by 2004-2005 ASCO President David Johnson, $\mathrm{MD}$, should be given credit for moving the quality agenda forward, and I am grateful to the very capable volunteers involved in this effort.

The Cancer Quality Alliance represents the most recent step forward in achieving ASCO's cornerstone mission of quality cancer care. The catalyzing efforts of ASCO volunteer Patricia Ganz, MD, and Ellen Stovall of the National Coalition of Cancer Survivors have resulted in a unique collaboration with the potential to appropriately align incentives and truly elevate the standard of practice so that cancer patients receive the quality cancer care they want and deserve.

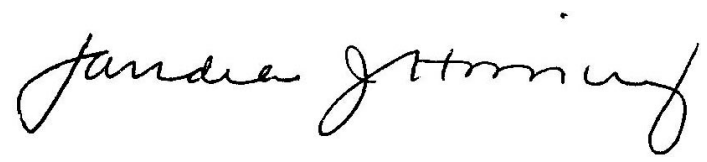

